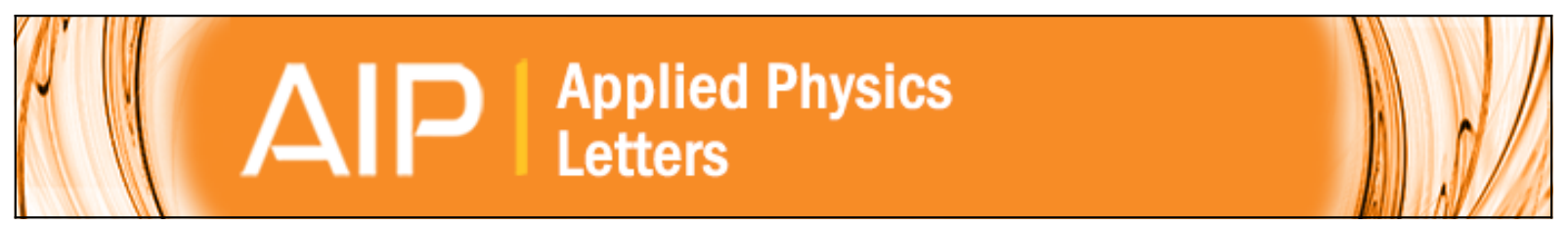

\title{
Spectral broadening in Brillouin imaging
}

Giuseppe Antonacci, Matthew R. Foreman, Carl Paterson, and Peter Török

Citation: Applied Physics Letters 103, 221105 (2013); doi: 10.1063/1.4836477

View online: http://dx.doi.org/10.1063/1.4836477

View Table of Contents: http://scitation.aip.org/content/aip/journal/apl/103/22?ver=pdfcov

Published by the AIP Publishing

\section{Articles you may be interested in}

Eliminating the broadening by finite aperture in Brillouin spectroscopy

Rev. Sci. Instrum. 82, 113110 (2011); 10.1063/1.3663072

Line Shapes in Brillouin Spectroscopy : the "platelet" Scattering Geometry

AIP Conf. Proc. 1290, 289 (2010); 10.1063/1.3517575

Brillouin imaging

Appl. Phys. Lett. 87, 061903 (2005); 10.1063/1.1999857

Spin-wave modes and line broadening in strongly coupled epitaxial $\mathrm{Fe} / \mathrm{Al} / \mathrm{Fe}$ and $\mathrm{Fe} / \mathrm{Si} / \mathrm{Fe}$ trilayers observed by Brillouin light scattering

J. Appl. Phys. 93, 3427 (2003); 10.1063/1.1554758

Debye temperature of erbium-doped yttrium aluminum garnet from luminescence and Brillouin scattering data J. Appl. Phys. 87, 1769 (2000); 10.1063/1.372090

\section{Asylum Research Atomic Force Microscopes}

Unmatched Performance, Versatility and Support

Modulus of Polymers

\& Advanced Materials

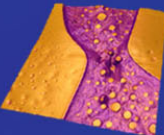

Coating Uniformity \& Roughness

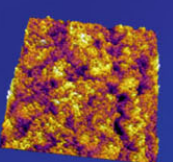

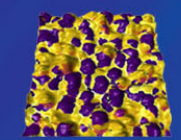

Nanoscale Conductivity \& Permittivity Mapping
Piezoelectrics

\& Ferroelectrics
+1 (805) 696-6466 sales@AsylumResearch.com www.AsylumResearch.com 


\title{
Spectral broadening in Brillouin imaging
}

\author{
Giuseppe Antonacci, ${ }^{1}$ Matthew R. Foreman, ${ }^{1,2}$ Carl Paterson, ${ }^{1}$ and Peter Török ${ }^{1, a)}$ \\ ${ }^{1}$ Blackett Laboratory, Department of Physics, Imperial College London, Prince Consort Road, \\ London SW7 2BZ, United Kingdom \\ ${ }^{2}$ Laboratory of Nanophotonics and Biosensing, Max Planck Institute for the Science of Light, \\ Günther-Scharowsky-Straße 1, 91058 Erlangen, Germany
}

(Received 27 September 2013; accepted 14 November 2013; published online 26 November 2013)

Brillouin microscopy is an emerging imaging modality that provides fundamental information about mechanical properties of media in a non-contact manner. To date, low numerical aperture (NA) optics have been used, due to noticeable angular broadening of the Brillouin spectrum at higher NAs. In this work, we investigate theoretically and experimentally the dependence of spectral broadening effects in Brillouin imaging on system NA, for both $90^{\circ}$ and $180^{\circ}$ scattering geometries. Lineshape deformations and broadening are found to be minimised in a backscattering geometry, hence paving the way for high resolution in-vivo mechanical imaging. (C) 2013 AIP Publishing LLC. [http://dx.doi.org/10.1063/1.4836477]

Brillouin scattering, first reported in $1922,{ }^{1}$ has since seen extensive use, for example, in material characterisation and sensing. ${ }^{2,3}$ Measurements of both the frequency shift of inelastically scattered photons and the linewidth of the resulting Brillouin peaks provide unique information regarding mechanical properties of materials, such as strain, temperature, stiffness, and elasticity constants. ${ }^{4-8}$ More recently, Brillouin spectroscopy has been extended from a point sampling technique into an imaging modality, ${ }^{9}$ heralding potential applications in cellular imaging and in-vivo diagnostics. Recent progress with virtually imaged phased arrays (VIPAs) ${ }^{10,11}$ has enabled a dramatic reduction of acquisition times. ${ }^{12}$ Realisation of a confocal Brillouin microscope and its application to biomechanical measurements of a mouse eye lens has recently been reported. ${ }^{13}$

Brillouin imaging has, to date, only been used with low numerical aperture (NA) microscope objectives. Primarily, this restriction arises in an attempt to limit spectral broadening of the Brillouin peaks, and hence loss of spectral resolution, associated with collection of photons over a wide range of scattering angles. ${ }^{14-17}$ Low NA lenses limit the achievable spatial resolution. Although a natural limit on the achievable spatial resolution is imposed by the acousto-optic interaction length (usually of the order of $1 \mu \mathrm{m}$ ), ${ }^{18}$ significant advances towards this fundamental limit can be made if higher NA optics can be employed. In this Letter, we investigate the inherent trade-off between spatial and spectral resolution in Brillouin imaging setups employing high NA lenses.

Before giving a rigorous treatment of spectral broadening arising from use of finite NA optics, it is insightful to initially consider a heuristic description. With reference to Figure 1, we consider light propagating in direction $\hat{\mathbf{k}}=(\sin \theta \cos \phi$, $\sin \theta \sin \phi, \cos \theta)$ incident upon a scattering medium. Upon inelastic scattering through an angle $\psi$, the scattered field propagates in direction $\hat{\mathbf{k}}^{\prime}=\left(\sin \theta^{\prime} \cos \phi^{\prime}, \sin \theta^{\prime} \sin \phi^{\prime}, \cos \theta^{\prime}\right)$. Conservation of energy and momentum dictate that the scattered photon is shifted in frequency by an amount

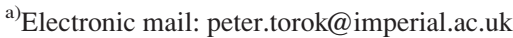

$$
\nu_{B}(\psi)=2 \nu_{0}(n / c) V \sin (\psi / 2),
$$

where $\nu_{0}$ is the frequency of the incident photon, $n$ is the refractive index of the medium, $c$ is the speed of light, and $V$ is the acoustic velocity in the scattering medium. ${ }^{18}$ The finite lifetime of acoustic phonons implies a frequency broadening such that the associated Brillouin spectral peak has a linewidth of

$$
\Delta \nu_{B}(\psi)=\frac{4 \eta \nu_{0}^{2} n^{2}}{\pi \rho c^{2}} \sin ^{2}(\psi / 2),
$$

where $\eta$ and $\rho$ are the viscosity and density of the material. ${ }^{18}$ The use of finite NA optics, however, implies the photons scattered over a range of $\psi=\cos ^{-1}\left[\hat{\mathbf{k}} \cdot \hat{\mathbf{k}}^{\prime}\right]$ all contribute to the observed signal. Accordingly, the Brillouin spectral line is formed from the incoherent superposition of many individual peaks with displaced maxima and differing widths. The total linewidth of the Brillouin peak is determined by the inherent Brillouin broadening $\Delta \nu_{B}$ and range of shifts $\nu_{B}$ present, however, typically $\Delta \nu_{B} \ll \nu_{B}$ such that we can neglect the former. Collection of photons scattered over a small range of scattering angles, $\delta \psi$, therefore gives rise to a range of Brillouin shifts, $\delta \nu_{B}$, which all contribute to the total observed peak, such that to first order the spectral broadening scales as ${ }^{19}$

$$
\left.\delta \nu_{B} \approx \frac{d \nu_{B}(\psi)}{d \psi}\right|_{\psi=\Psi} \delta \psi \propto \cos (\psi / 2) \delta \psi
$$

where $\Psi$ is the average scattering angle. Noting that

$$
\left.\frac{d \nu_{B}(\psi)}{d \psi}\right|_{\psi=\pi}=0
$$

suggests that the spectral broadening effects arising from use of finite NA optics are minimised in a back-scattering geometry.

We note that the incident and scattered directions can be defined by positions on the surface of the Gaussian reference spheres associated with the illumination and collection 


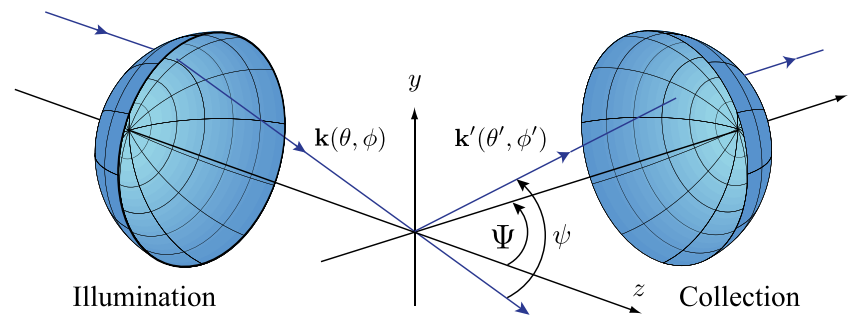

FIG. 1. Schematic of scattering geometry showing the Gaussian reference spheres for illumination and collection lenses. The direction of incident and scattered photons are defined by the wave-vectors $\mathbf{k}(\theta, \phi)$ and $\mathbf{k}^{\prime}\left(\theta^{\prime}, \phi^{\prime}\right)$, respectively.

lenses (Figure 1). ${ }^{20}$ Specifically, we must then integrate the spectral profiles, $s(\nu, \psi)$, obtained for all possible scattering angles over the associated solid angles

$$
S(\nu)=\int_{\Omega} \int_{\Omega^{\prime}} s(\nu, \psi) d \Omega d \Omega^{\prime},
$$

where the inherent spectrum of acoustic waves propagating in liquids is known to be well described by a Lorentzian profile of the form

$$
s(\nu, \psi)=\frac{\left(\Delta \nu_{B}(\psi) / 2\right)^{2}}{\left(\nu-\nu_{0} \pm \nu_{B}(\psi)\right)^{2}+\left(\Delta \nu_{B}(\psi) / 2\right)^{2}},
$$

with the \pm sign taken for the anti-Stokes and Stokes components, respectively. ${ }^{21}$ Equations (5) and (6) give the total Brillouin spectrum arising from the detection of acoustic phonons with finite NA. We shall now investigate the two limiting scattering geometries, $\Psi=90^{\circ}$ and $180^{\circ}$, using numerical evaluation of Eq. (5).

Figure 2 shows an example calculation for Brillouin scattering (via an anti-Stokes process) from a liquid benzene sample $\left(\eta=0.652 \times 10^{-2} \mathrm{~g} \mathrm{~cm}^{-1} \mathrm{~s}^{-1}, \rho=0.876 \mathrm{~g} \mathrm{~cm}^{-3}\right.$, $n=1.5$ and $V=1276.4 \mathrm{~m} \mathrm{~s}^{-1}$ ) in which the spectral intensity (normalised to the Rayleigh peak) is shown on a logarithmic scale, as a function of the illumination and collection NA (which were taken to be equal). It should be noted that the total collected power scales as the product of the illumination and collection solid angles, i.e., $\Omega \Omega^{\prime}$, where $\Omega^{(\prime)}=2 \pi\left(1-\cos \alpha^{(\prime)}\right)$, as depicted in the inset of Figure 2 . For reference, the theoretical Brillouin shifts for a $90^{\circ}$ and $180^{\circ}$ scattering geometry with NA $\approx 0$ are $\nu_{B}^{90}=4.48$ and $\nu_{B}^{180}=6.19 \mathrm{GHz}$, respectively. For the $90^{\circ}$ geometry, the Brillouin peak is subject to a dramatic broadening as the NA is increased. Furthermore, the Brillouin peak noticeably changes in shape from its inherent Lorentzian profile leading to a substantial blueshift in the central frequency with respect to $\nu_{B}^{90}$. This is clearly seen in Figure 3, which shows an overall displacement of roughly $18 \%$ in the peak position in the frequency domain. Naive use of Eq. (1) would therefore lead to significant errors in determination of the acoustic velocity of phonons, and thus of the elasticity moduli of the material in any realistic experimental study. Figure 3 also illustrates the broadening of the FWHM of the Brillouin peak as a function of NA. An order of magnitude increase in $\Delta \nu_{\text {tot }}$ over a full aperture is evident, implying a degradation of overall spectral resolution. The Rayleigh peak remains constant in width and centred at the incident frequency $\nu_{0}$ for all NA as expected.

For the $180^{\circ}$ backscattering geometry, however, a narrower Brillouin peak is obtained. Again the Rayleigh peak is subject to neither spectral broadening nor shifts as the system NA is varied. In Figure 3, the Brillouin peak FWHM $\Delta \nu_{\text {tot }}$ is seen to remain below $1 \mathrm{GHz}$ even for NAs close to unity. Shifts of the apparent Brillouin peak are also reduced by approximately an order of magnitude. Similar results are also found when assuming a liquid methanol sample.

To verify the preceding results experimentally, we implemented a Brillouin microscope setup in both $90^{\circ}$ and $180^{\circ}$ scattering geometries. Initial setup and system verification was performed by measuring the Brillouin spectra of assorted liquids at low NA. Figure 4 shows the optical setup for the $90^{\circ}$ geometry. The beam emitted by a single longitudinal mode laser $(\lambda=561 \mathrm{~nm})$ is expanded to $25 \mathrm{~mm}$ to fill the pupil of an achromatic lens $L_{\text {ill }}(f=30 \mathrm{~mm})$, which focuses the light from air into a glass cuvette filled with the liquid sample. A Glan-Thompson (GT) polariser and a halfwave plate ensure s-polarised light at the sample plane. The scattered light is collected by a second achromatic lens $L_{\mathrm{col}}$ $(f=30 \mathrm{~mm})$, is spatially filtered and then dispersed (in angle) by a custom built VIPA. The VIPA is similar in design to a typical Fabry-Perot etalon, except for an additional antireflection coated entrance window through which focused light enters. This design helps to minimise insertion losses,

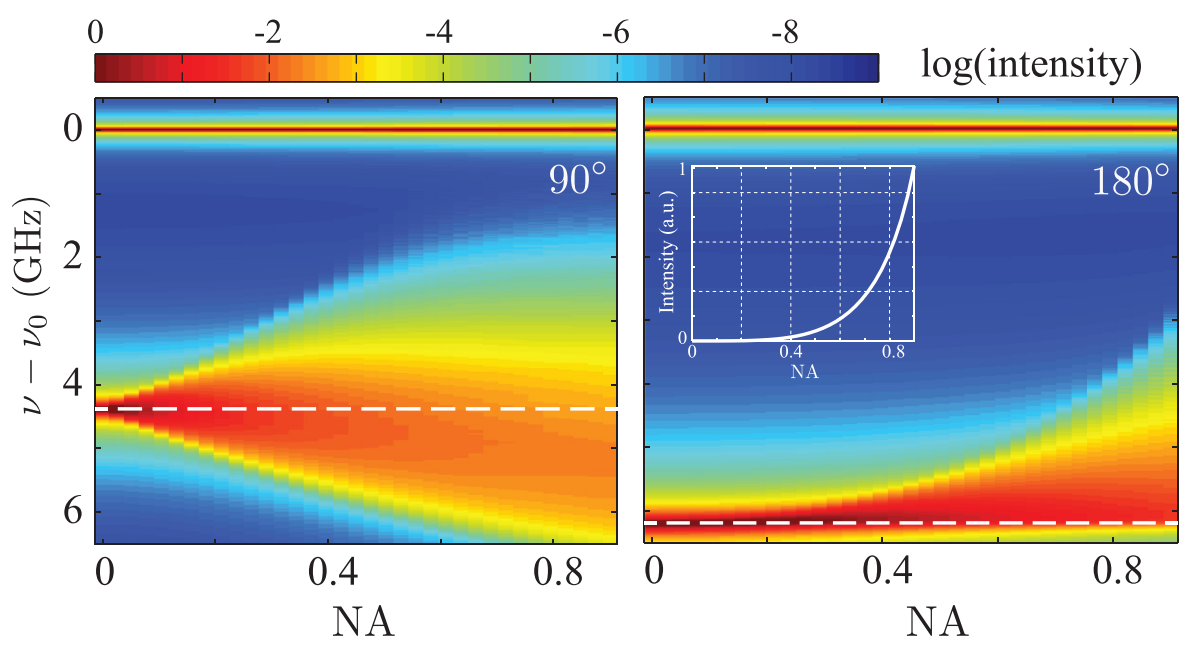

FIG. 2. Logarithmic spectral intensity as a function of NA for scattering from liquid benzene in a $90^{\circ}$ (left) and $180^{\circ}$ (right) geometry. The spectrum is normalised to the peak Rayleigh intensity at each NA. White dashed lines show the theoretical position of the Brillouin peak for $\mathrm{NA}=0$. (Inset) Total integrated intensity as a function of NA. 

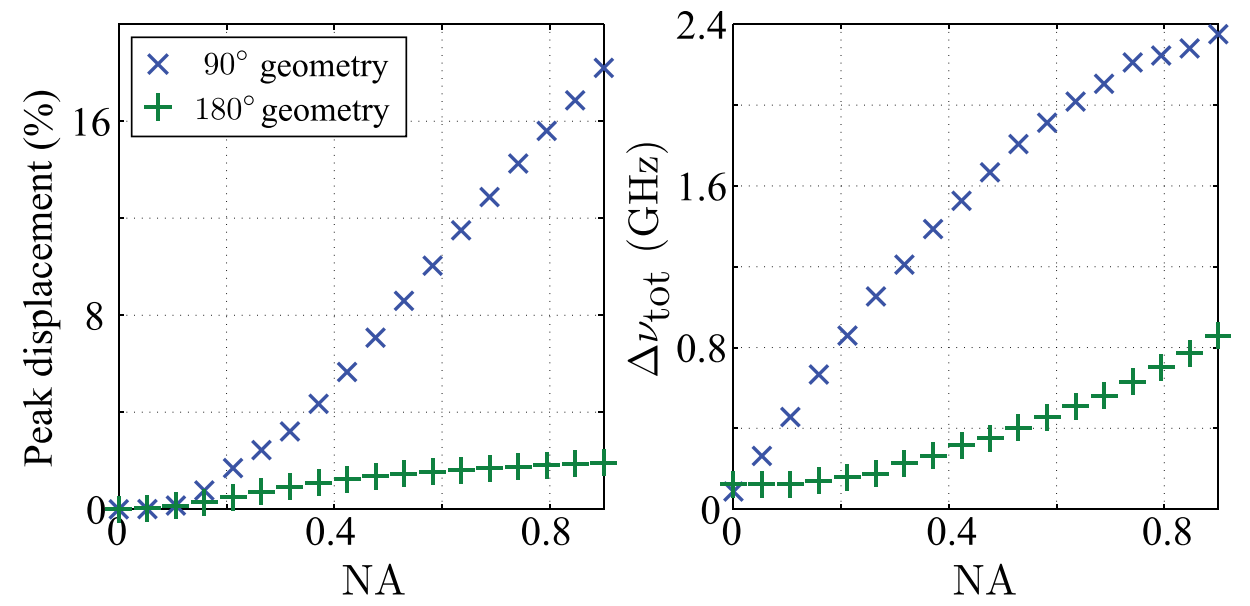

FIG. 3. Relative shift of Brillouin peak from $\nu_{B}^{90,180}$ (left) and FWHM (right) as a function of the NA (left). therefore allowing greater extinction ratios and faster acquisition times to be achieved. ${ }^{10}$ The VIPA has a measured spectral resolution of $\delta \nu=(438 \pm 5) \mathrm{MHz}$, a free spectral range of $\Delta \nu=(39.5 \pm 0.5) \mathrm{GHz}$ and a finesse of $\mathcal{F}=90.2$ \pm 0.5 . The output field of the VIPA is Fourier transformed so as to spatially disperse the Brillouin spectrum, which is then recorded by a sCMOS camera with an integration period of a few seconds. To allow high precision alignment, a second CCD camera is used to image the scattering volume and to match it with a reference beam sent through the collection arm. The interference pattern recorded by the sCMOS camera is processed for background subtraction and Lorentzian fitting of the resulting spectral peaks. Figure 5 shows the calibration spectra acquired at low NA for different liquid samples after processing. Experimental data points for methanol are also plotted for comparison. Brillouin frequency shifts relative to the central Rayleigh peak are in good agreement with the theoretical values, as illustrated in Table I.

Brillouin spectra of liquid benzene for a range of NAs were recorded by varying the radius $r_{l}$ of the expanded laser beam by means of a diaphragm $D^{\prime}$ placed behind $L_{\mathrm{ill}}$. The $\mathrm{NA}$ of the collection arm was held constant at $\mathrm{NA}^{\prime}=0.13$. The same procedure was applied in a backscattering arrangement where the illumination and collection were performed by the same lens. In this case, a dark-field configuration was used to minimise detection of surface reflections. Specifically, an annular illumination was used whilst the scattered radiation was collected through the central region of the pupil.

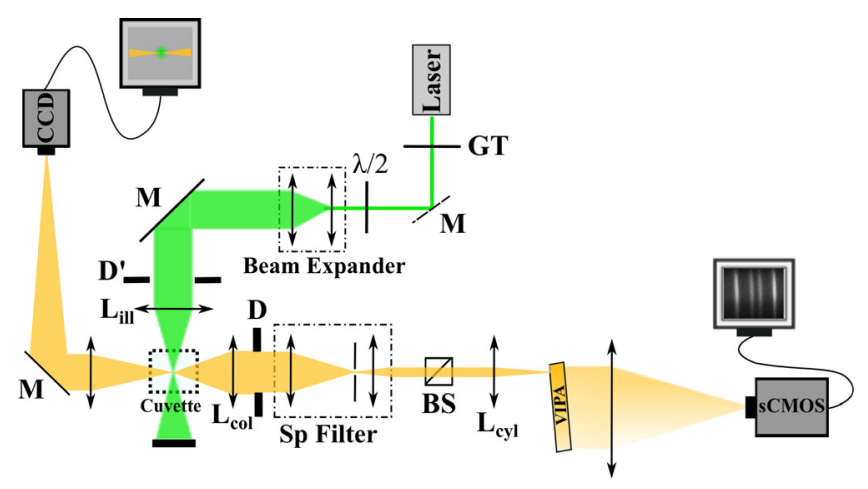

FIG. 4. Optical setup for a $90^{\circ}$ scattering geometry.
Figure 6 shows the variation of the measured antiStokes Brillouin peak linewidths $\Delta \nu_{\text {inst }}$ for a range of NAs for both scattering geometries. Theoretical values, depicted by the solid black lines, were determined by calculating the integrated spectrum of liquid benzene, obtained from Eq. (5), convolved with the instrumental function of the VIPA. Following Oliver ${ }^{14}$ and LaMacchia, ${ }^{15}$ the instrumental function was taken as a Lorentzian function with FWHM matching the elastic Rayleigh peak. This method requires the spectral bandwidth of the laser to be small, as is the case for

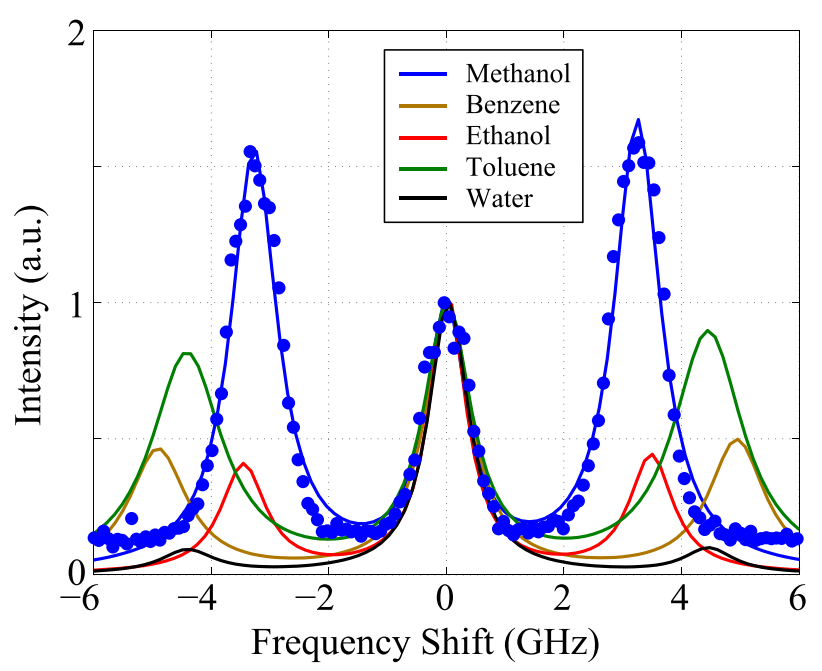

FIG. 5. Fitted Brillouin spectra (solid lines) for different liquids obtained in a $90^{\circ}$ scattering geometry with $\mathrm{NA}=\mathrm{NA}^{\prime}=0.03$. Individual spectra have been normalised to the central Rayleigh peak. Experimental data points for methanol (dots) are also shown for comparison. Each spectrum was acquired using a $2 \mathrm{~s}$ exposure time and a laser power of $10 \mathrm{~mW}$ at the sample plane.

TABLE I. Experimental and theoretical values of the Brillouin frequency shift for different liquids as found from spectra acquired in a $90^{\circ}$ scattering geometry at room temperature with $\mathrm{NA}=\mathrm{NA}^{\prime}=0.03$.

\begin{tabular}{lccr}
\hline \hline & $\nu_{B} \exp .(\mathrm{GHz})$ & $\nu_{B}$ theo. $(\mathrm{GHz})$ & $V(\mathrm{~m} / \mathrm{s})$ \\
\hline Methanol & $3.26 \pm 0.06$ & 3.39 & 973.8 \\
Benzene & $4.92 \pm 0.07$ & 4.38 & 1301.1 \\
Ethanol & $3.47 \pm 0.04$ & 3.48 & 1012.1 \\
Toluene & $4.43 \pm 0.08$ & 4.26 & 1174.7 \\
Water & $4.43 \pm 0.08$ & 4.41 & 1341.5 \\
\hline
\end{tabular}



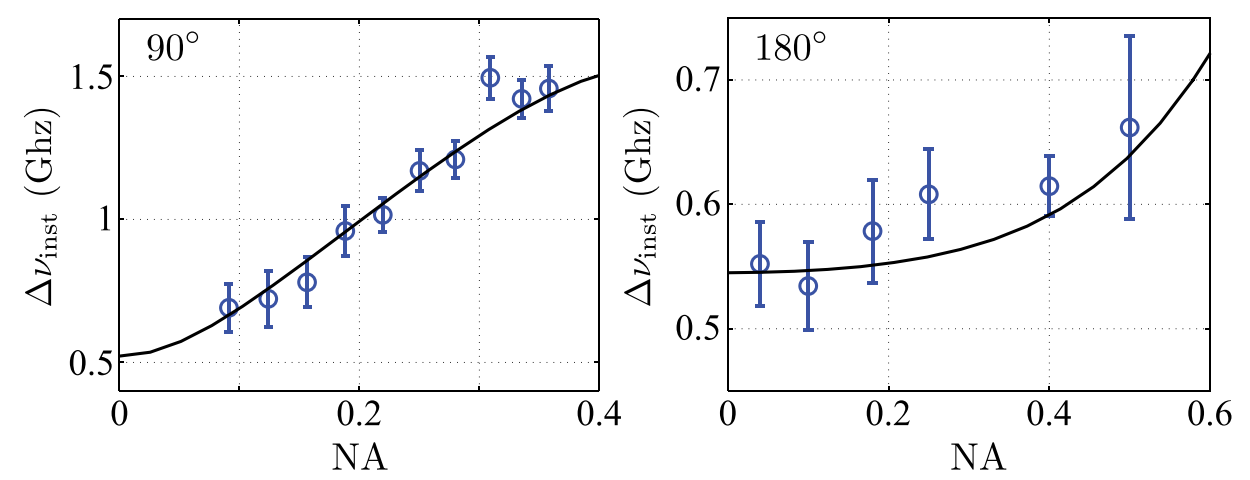

FIG. 6. Brillouin peak linewidth measurements (blue circles, with accompanying error bars) as a function of the NA for $\Psi=90^{\circ}$ (left) and $\Psi=180^{\circ}$ (right). Black solid curves depict theoretical predictions. a single longitudinal mode laser. From Figure 6, the resulting spectral linewidth is seen to increase by $\sim 1 \mathrm{GHz}$ for $\Psi=90^{\circ}$ whilst it does not vary significantly for $\Psi=180^{\circ}$. Experimental results show good agreement with theory. The superiority of the backscattering geometry is clearly seen, suggesting that Brillouin peaks can be localised and resolved for high NA systems utilising this geometry.

In summary, in this Letter, we have analysed the effects of finite instrumental apertures on experimental Brillouin spectra, with a view to high NA Brillouin imaging. By integrating over finite pupil geometries we have shown that broadening effects could be minimised by use of a backscattering geometry. Furthermore, deformation of the Lorentzian line shape of the Brillouin peak was shown to arise from use of high NAs such that the peak maximum is shifted relative to the low NA case. These effects were again shown to be minimised in a backscattering geometry. Experimental verification of these predictions followed using a $90^{\circ}$ and $180^{\circ}$ Brillouin dark-field microscope setup. Our results show that whilst the common practice of use of low NA lenses in a $90^{\circ}$ scattering geometry is necessary to minimise angular broadening and spectral distortion, these effects are far less apparent in a backscattering geometry. High NA (NA >0.5) lenses can, therefore, be used with limited consequences in such a geometry. Our analysis can further provide accurate evaluation of suitable fitting functions since spurious frequency shifts can be obtained when fitting using a symmetric lineshape. ${ }^{16}$ Ultimately, Brillouin imaging with both high spatial and spectral resolution, hitherto thought unfeasible, is hence a realistic proposition.

${ }^{1}$ L. Brillouin, Ann. Phys. 17, 88 (1922).

${ }^{2}$ R. J. Angel, J. M. Jackson, H. J. Reichmann, and S. Speziale, Eur. J. Mineral. 21, 525 (2009).

${ }^{3}$ T. R. Parker, M. Farhadiroushan, V. A. Handerek, and A. J. Roger, IEEE Photon. Technol. Lett. 9, 979 (1997).

${ }^{4}$ J. Smith, A. Brown, M. DeMerchant, and X. Bao, Appl. Opt. 38, 5372 (1999).

${ }^{5}$ G. Scarcelli, P. Kim, and S. H. Yun, Biophys. J. 101, 1539 (2011).

${ }^{6}$ S. A. Lee, D. A. Pinnick, S. M. Lindsay, and R. C. Hanson, Phys. Rev. B 34, 2799 (1986).

${ }^{7}$ S. K. Satija and C. Wang, Solid State Commun. 28, 617 (1978).

${ }^{8}$ K. J. Koski, P. Akhenblit, K. McKiernan, and J. L. Yarger, Nature Mater. 12, 262-267 (2013).

${ }^{9}$ K. J. Koski and J. L. Yarger, Appl. Phys. Lett. 87, 061903 (2005).

${ }^{10}$ M. Shirasaki, Fujitsu Sci. Tech. J. 35, 113 (1999).

${ }^{11}$ S. A. Diddams, L. Hollberg, and V. Mbele, Nature 445, 627 (2007).

${ }^{12}$ G. Scarcelli and S. H. Yun, Opt. Express 19, 10913 (2011).

${ }^{13} \mathrm{G}$. Scarcelli and S. H. Yun, Nat. Photonics 2, 39 (2008).

${ }^{14}$ W. F. Oliver, C. A. Herbst, S. M. Lindsay, and G. H. Wolf, Rev. Sci. Instrum. 63, 1884 (1992).

${ }^{15}$ J. H. W. Leidecker and J. T. LaMacchia, J. Acoust. Soc. Am. 43, 143 (1968).

${ }^{16}$ M. G. Beghi, F. Di Fonzo, S. Pietralunga, C. Ubaldi, and C. E. Bottani, Rev. Sci. Instrum. 82, 053107 (2011).

${ }^{17}$ M. G. Beghi, A. G. Every, and P. V. Zinin, Ultrasonic Nondestructive Evaluation: Engineering and Biological Material Characterization (CRC Press, Boca Raton, 2004).

${ }^{18}$ M. Damzen, Stimulated Brillouin Scattering: Fundamentals and Applications (IOP Publishing, Bristol, 2003).

${ }^{19}$ E. Rat, M. Foret, G. Massiera, R. Vialla, M. Arai, R. Vacher, and E. Courtens, Phys. Rev. B 72, 214204 (2005).

${ }^{20}$ M. R. Foreman and P. Török, J. Mod. Opt. 58, 339 (2011).

${ }^{21}$ H. G. Danielmeyer, J. Acoust. Soc. Am. 47, 151 (1970). 